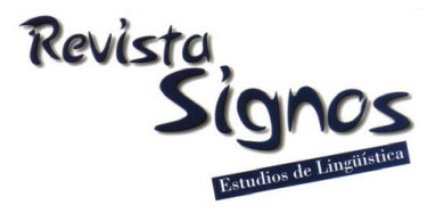

\title{
Los nombres rotuladores: Un estudio de los rótulos cohesivos en artículos de investigación en inglés y español${ }^{1}$
}

\section{Labelling nouns: A study of cohesive labels in research articles in English and Spanish}

\section{Verónica L. Muñoz}

Universidad NACIONAL DE Río CuARTo ARGENTINA

vmunoz@hum.unrc.edu.ar

\author{
Guiomar E. Ciapuscio \\ UNIVERSIDAD DE BUENOS AIRES/CONICET \\ ARGENTINA \\ gciapusc@gmail.com
}

\section{Recibido: 20-V-2018 / Aceptado: 05-XII-2018 \\ DOI: $10.4067 /$ S0718-09342019000200688}

\section{Resumen}

El propósito de este trabajo es presentar un estudio lingüístico-descriptivo de un grupo de nombres, que llamamos 'rotuladores', basado en un corpus de artículos de investigación de las ciencias agrarias escritos en inglés y en español. Nos interesa profundizar en los principales rasgos morfológicos, semánticos y pragmáticos de estos nombres rotuladores, que funcionan como núcleo nominal de los rótulos cohesivos, y presentar una propuesta para su ordenamiento semántico-conceptual. Los rótulos cohesivos son sintagmas nominales empleados como recursos conectivos que organizan y dan unidad a los textos al encapsular y rotular conceptualmente segmentos textuales, establecer enlaces extraoracionales y textualizar la función organizativa e interpersonal de los textos (por ej., this complex process, la misma tendencia). Los resultados indican que los nombres rotuladores son principalmente nominalizaciones deverbales, poseen significados generales, representan entidades de segundo y tercer orden, pueden tener carga valorativa para comunicar actitud y evaluación, y pueden ser de naturaleza metadiscursiva para orientar la organización del texto. Estas propiedades convierten a los nombres rotuladores en componentes centrales de los rótulos cohesivos en inglés y en español.

Palabras Clave: Nombres rotuladores, rótulos cohesivos, morfología, semántica, pragmática. 


\begin{abstract}
The purpose of this study is to report on a descriptive-linguistic analysis of a group of nouns, here referred to as 'labelling nouns', based on a corpus of agriculture research articles written in English and in Spanish. Our aim is to analyze the main morphological, semantic, and pragmatic features of these labelling nouns, used as the head of cohesive labels, and to present a proposal for their semantic and conceptual categorization. Cohesive labels are nominal phrases used as connective resources that organize and give unity to texts by encapsulating and labelling textual segments conceptually, establishing intersentential links and textualizing the organizing and interpersonal function of texts (e.g. this complex process, la misma tendencia). The results indicate that labelling nouns are mainly verbal nominalizations, have general meanings, represent second and third order entities, can have a value component to communicate attitude and evaluation, and can be metadiscursive to organize texts. These features make labelling nouns central elements of cohesive labels in English and in Spanish.
\end{abstract}

Key Words: Labelling nouns, cohesive labels, morphology, semantics, pragmatics.

\title{
INTRODUCCIÓN
}

Los nombres generales de Halliday y Hasan (1976) impulsaron una serie de estudios teórico-descriptivos sobre un grupo de nombres caracterizados por poseer significados generales e inespecíficos (por ej., cosa, asunto), aquí denominados 'nombres rotuladores'2. Se han propuesto distintas categorías que han recibido diversas denominaciones, con puntos de convergencia y diferencias conceptuales: 'nombres generales' (Halliday \& Hasan, 1976; Mahlberg, 2005), 'nombres anafóricos' (Francis, 1986), 'nombres portadores' (Ivanic, 1991), 'nombres inespecíficos' (Winter, 1992), 'nombres cápsula' (Hunston \& Francis, 2000; Schmid, 2000, 2018), 'nombres señalizadores' (Flowerdew, 2003, 2009; Flowerdew \& Forest, 2015), 'etiquetas discursivas’ (López Samaniego, 2011), ‘nombres metadiscursivos’ (Jiang \& Hyland, 2016, 2017) y 'encapsuladores nominales infieles' (López Samaniego, 2018). La variedad terminológica se debe a que cada categoría se define conceptualmente desde distintas perspectivas. Los estudios han concluido que, en los distintos corpus analizados, los nombres rotuladores tienden a ser frecuentes en los textos escritos (Francis, 1986, 1994; López Samaniego, 2011; Benítez Castro, 2013; Flowerdew \& Forest, 2015), en los géneros académicos (Ivanic, 1991; Flowerdew, 2003, 2009, 2015; Benítez Castro \& Thompson, 2015; Flowerdew \& Forest, 2015), con una mayor frecuencia en los artículos de investigación (Flowerdew \& Forest, 2015).

Independientemente de las denominaciones, las propiedades semánticas de estos nombres los hacen productivos para crear cohesión, dado que tienen la capacidad de encapsular y rotular conceptualmente el contenido de segmentos textuales, como las oraciones y los párrafos. Por esta razón son de gran relevancia, pues en determinados contextos y funciones, pueden realizar dos operaciones discursivas simultáneamente: sintetizan el contenido de fragmentos y los categorizan de una determinada manera. 
Si bien las propiedades de condensación y rotulación de estos nombres son señaladas en la literatura (Francis, 1986; Ivanic, 1991; Hunston \& Francis, 2000; Schmid, 2000, 2018; Flowerdew, 2003, 2009, 2015; López Samaniego, 2011, 2018; Flowerdew \& Forest, 2015), no se ha privilegiado su capacidad de conectar partes sintácticamente independientes, a excepción de algunos autores (Halliday \& Hasan, 1976; Francis, 1986) que se centran puntualmente en los nombres que encapsulan segmentos fuera de los límites de la oración. Es decir, la mayoría de las conceptualizaciones abarcan distintos usos de los nombres rotuladores, tales como la cohesión, organización y evaluación (Halliday \& Hasan, 1976; Francis, 1986), encapsulación y rotulación (Ivanic, 1991; Schmid, 2000, 2018; Flowerdew \& Forest, 2015; López Samaniego, 2011, 2018), señalización (Flowerdew, 2003, 2009) y función metadiscursiva (Flowerdew, 2015; Jiang \& Hyland, 2016, 2017). De todos estos usos, los nombres rotuladores tienden a emplearse cohesivamente como mecanismos que señalan hacia afuera de los límites de la oración (Flowerdew \& Forest, 2015). Por esta razón, el estudio de los nombres rotuladores como enlaces extraoracionales merece atención. A este espacio de investigación, precisamente, esperamos poder contribuir con nuestro estudio.

Al ser nombres que se definen funcionalmente (Schmid, 2000, 2018), en este trabajo estudiamos los nombres rotuladores que aparecen como núcleo nominal de sintagmas nominales que hemos denominado 'rótulos cohesivos' (RRCC), basándonos en las categorías de 'rótulos' de Francis (1994) y de 'etiquetas discursivas' de López Samaniego $(2013,2015)$. Los RRCC, aunque conceptualmente muy próximos a los rótulos y las etiquetas discursivas, se distinguen por ser sintagmas nominales que realizan simultáneamente tres operaciones básicas para lograr la unidad y organización de los textos: sintetizan y rotulan el contenido de fragmentos textuales, establecen cohesión y enlazan partes del texto como nexos extraoracionales que marcan transiciones. Por su función conectiva y su capacidad de categorizar segmentos del texto, los rótulos cohesivos funcionan como enlaces y señalizadores textuales, de carácter anafórico o catafórico, que permiten conectar fragmentos textuales, al recapitular (1) o anticipar (2) información para la coherencia y organización del texto:

(1) El número de plántulas fue mayor en los tratamientos que incluian originalmente rizomas.

Este hecho puede atribuirse a que las plantas provenientes de rizomas florecieron un mes antes que las originadas de semillas y por lo tanto habrían anticipado su aporte de semillas al suelo.

(2) Simulating steady-state systems with extra feed was a two-step process. Firstly, the amended monthly HAR values (Appendix A) were entered into the Base UDDER model, and the system was re-simulated in the absence of any further management adjustments. In all instances, this resulted in increases in average farm pasture cover and BCS which were considered to render the system unsustainable, sometimes markedly so. (. . .).

The second step was then to amend the simulations incorporating the extra feed to bring the key system indicators of pasture cover and BCS back into line with the Base simulation. This 
entailed altering one or more of the following production inputs or management policies: amount and timing (...)

Como se observa en los ejemplos, los RRCC son un recurso fundamental para desplegar textos altamente retóricos, como lo es el artículo de investigación. En el presente trabajo estudiamos una muestra de 82 artículos de investigación de las ciencias agrarias escritos en inglés y en español. Nos centramos en las ciencias agrarias dado que el tema bajo estudio, según nuestro conocimiento, no ha sido explorado en esta disciplina. Nos enfocamos en un género y disciplina específica puesto que se han demostrado variaciones en el uso de los nombres rotuladores en diferentes contextos (Charles, 2003; Schmid, 2000, 2018; Flowerdew, 2015; Flowerdew \& Forest, 2015). Los objetivos de nuestro trabajo son:

1. Identificar y realizar un inventario de los nombres rotuladores que funcionan como núcleo de los RRCC en las dos lenguas;

2. Describir desde el punto de vista formal, semántico y pragmático el conjunto de nombres rotuladores y presentar una sistematización consistente con los datos.

\section{Marco teórico}

El antecedente teórico de los nombres rotuladores tiene sus raíces en el trabajo de Halliday y Hasan (1976). Desde la perspectiva de la gramática sistémica funcional, los autores pusieron en evidencia la existencia de un grupo de nombres que denominaron 'nombres generales', que se distinguen semánticamente de otros nombres dado que tienen significados generales y representan clases amplias de entidades: por ej., cosa, persona, lugar, idea, asunto. Lo interesante de estos nombres es que al representar grupos de entidades operan cohesivamente y pueden remitir no sólo a sintagmas nominales sino también a segmentos del texto de diferente extensión. En combinación con determinantes (por ej., los demostrativos y el artículo definido), funcionan al igual que una forma pronominal que establece cohesión con otras entidades del texto. En estos casos, todo el sintagma nominal funciona como una expresión anafórica cuya interpretación es posible sólo en relación con lo que se ha mencionado anteriormente. Se definen como una categoría situada a medio camino entre la cohesión léxica y la cohesión gramatical.

El trabajo de Halliday y Hasan (1976) sobre los nombres generales sentó precedentes para el desarrollo de una línea de investigación que se ha ocupado de caracterizar este grupo de nombres a partir de su ocurrencia en diferentes contextos de uso. A lo largo de 40 años de trabajo académico la noción se ha expandido, redefinido y ramificado en diferentes direcciones (Winter, 1977, 1992; Francis, 1986, 1994; Ivanic, 1991; Hunston \& Francis, 2000; Schmid, 2000, 2018; Flowerdew, 2003, 2009, 2015; Mahlberg, 2005; López Samaniego, 2011, 2013, 2015, 2018; Flowerdew \& 
Forest, 2015; Jiang \& Hyland, 2016, 2017). Esta es la línea de investigación en que situamos teóricamente nuestro estudio y a la cual esperamos aportar con él.

Existen ciertos puntos conceptuales en común entre las categorías propuestas en la literatura: el principal es la caracterización semántica de los nombres, definidos como generales e inespecíficos. Las distintas conceptualizaciones coinciden en privilegiar el punto de vista semántico como el criterio central para su categorización como grupo. Todos los autores los caracterizan como palabras con significados generales o inespecíficos que se definen y se precisan en el contexto en que son usados, sea este el contexto discursivo, el de situación, el contexto cultural, o incluso la información compartida entre los interlocutores. Al respecto, Ivanic (1991) establece una diferencia entre el significado constante, estable, e inherente de los nombres, que aparece en los diccionarios, y los significados variables y transitorios que difieren según cada contexto. A modo de ejemplo, el nombre ventaja, definido como 'excelencia o condición favorable que alguien o algo tiene' (RAE), denota una clase de cualidad o atributo adscribible a un conjunto infinito de estados, fenómenos, condiciones, experiencias y situaciones, formada por aquellas situaciones que supongan una condición favorable. Por esta razón, dicho significado constante e inespecífico se precisa y varía en relación con la información que se recupera en cada contexto. El vacío semántico (Flowerdew \& Forest, 2015; Schmid, 2000) que debe completarse a partir del contexto específico de ocurrencia es precisamente la propiedad que explica el potencial de este grupo de nombres para funcionar como cápsulas conceptuales o rótulos cuyo contenido se concreta mediante la relación conceptual con la información provista por el contexto.

\section{Marco metodológico}

\subsection{Corpus}

En una primera instancia se construyeron dos corpus especializados (Sinclair, 1991; Hunston, 2002) y paralelos (Connor \& Moreno, 2005; Moreno, 2008) de artículos de investigación de las ciencias agrarias en inglés (199 textos, 1.074 .614 palabras) y español (199 textos, 598.601 palabras), publicados entre los años 2012-2007 en cuatro revistas científicas internacionales y cuatro revistas científicas argentinas ${ }^{3}$. Se consideraron principios de la lingüística de corpus (Sinclair, 1991, 2005; Biber, 2008) y de la retórica contrastiva (Connor \& Moreno, 2005) para garantizar sistematicidad y comparabilidad entre el inglés y el español. Ambos corpus sirvieron de base para extraer de manera rigurosa una muestra de 82 artículos de investigación (41 textos y 228.724 palabras en inglés, 41 textos y 126.282 palabras en español). Se eligieron los artículos publicados en el año 2011, una decisión metodológica que se tomó arbitrariamente como criterio de selección. 


\subsection{Diseño de investigación y procedimiento metodológico}

Realizamos un estudio lingüístico descriptivo. Se identificaron y analizaron los nombres rotuladores presentes en los RRCC identificados manualmente (sin asistencia de software) en la muestra de 82 artículos. Se realizó una descripción morfológica, semántica y pragmática de los nombres, a fin de sistematizar sus significados y usos en los artículos de investigación.

Para identificar los RRCC se emplearon tres criterios, basados en las características funcionales que los definen como unidad encapsuladora y rotuladora, a saber: sintetizan y encapsulan la información de fragmentos textuales, rotulan el contenido de los fragmentos encapsulados y funcionan como elementos cohesivos que enlazan partes del texto más allá de los límites oracionales.

En cuanto al primer criterio, los segmentos encapsulados por los RRCC debían ser fragmentos del texto, definidos en este estudio como unidades mayores a una palabra, una secuencia de palabras o un sintagma nominal. Con respecto a los sintagmas nominales, cabe aclarar que decidimos considerar como unidad mínima encapsulada sintagmas nominales complejos con estructura predicativa (López Samaniego, 2011, 2013, 2015), siempre que la estructura predicativa no contuviera la información condensada o encapsulada por el nombre rotulador. Asimismo, incluimos casos de sintagmas nominales de estructura compleja que contienen varias frases integradas con un alto grado de contenido informativo (Biber, Johansson, Leech, Conrad \& Finegan, 1999).

Respecto del segundo criterio, rotulación, el vínculo entre el RC y el segmento encapsulado debía ser una relación semántico-pragmática establecida específicamente en el co-texto. Este criterio excluye relaciones cohesivas determinadas por vínculos semánticos, de diversa naturaleza, ya supuestos en el sistema léxico de la lengua (Lyons, 1977; Cruse, 1986, 2000, 2006), tales como repetición exacta de una palabra y otras formas de reiteración (Halliday \& Hasan, 1976).

Con respecto al tercer criterio de identificación, los fragmentos textuales encapsulados por los RRCC debían encontrarse fuera de los límites de la oración que contenía el RC. Para determinar la función cohesiva de los RRCC como enlaces extraoracionales, en este estudio consideramos la oración ortográfica como unidad de análisis, al igual que en otros trabajos relacionados a la temática que abordamos en nuestra investigación (Halliday \& Hasan, 1976; Winter, 1992; Álvarez de Mon y Rego, 1999; Sinclair, 2004).

Para la caracterización morfológica nos basamos en el estudio lexicográfico de los nombres, por un lado, y en obras clásicas de morfología (Huddleston, 1984; Lacuesta \& Bustos Gisbert, 1999; Varela Ortega \& Piera, 1999; RAE, 2009), por el otro. La descripción semántica de los nombres se orientó en tratados clásicos de la semántica 
(Lyons, 1977; Cruse, 2000, 2006), en gramáticas descriptivas del inglés y el español (Quirk, Greenbaun, Leech \& Svartvik, 1985; Bosque, 1999; RAE, 2009) y, además, en el enfoque de la gramática sistémica funcional (Halliday, 1994a; Halliday \& Matthiessen, 2014). Agrupamos los nombres rotuladores a partir de sus significados y utilizamos categorías existentes en la literatura (Francis, 1986, 1994; Schmid, 2000, 2018; Flowerdew \& Forest, 2015) que sirvieron de base a la sistematización que proponemos en este estudio. Por último, para la descripción pragmática se recurrió a la gramática sistémica funcional (Matthiessen, 1995; Halliday \& Matthiessen, 2014) y a trabajos sobre el concepto de metadiscurso (Van de Kopple, 1985; Crismore \& Farnsworth, 1990; Fandrych \& Graefen, 2002).

\section{Resultados y discusión}

Identificamos una amplia variedad de nombres que funcionan como núcleo de los rótulos cohesivos para encapsular y conceptualizar fragmentos textuales. En los 595 RRCC identificados, aparecen 159 nombres rotuladores (por ej., phenomenon, rationale, premisa, problema). Esto pone en evidencia la diversidad de nombres que pueden desempeñar el papel de nombres rotuladores en el contexto del artículo de investigación de las ciencias agrarias en inglés y en español. De los 159 nombres identificados, aproximadamente el 45\% aparece únicamente en los RRCC en inglés, el $27 \%$ aparece solamente en los RRCC en español, y el 28\% se advierte en los RRCC de ambas lenguas (Tabla 1). La lista completa de los nombres identificados se presenta en el Anexo. No es posible establecer comparaciones con estudios previos, puesto que estos se han enfocado en diversas funciones y entornos sintácticos y han analizado los nombres rotuladores en distintos géneros y corpus. Los pocos trabajos sobre nombres rotuladores utilizados como enlaces extraoracionales (Francis, 1986, 1994; Halliday \& Hasan, 1976) no proporcionan datos cuantitativos.

Tabla 1. Nombres rotuladores identificados. Números absolutos y porcentajes.

\begin{tabular}{|c|c|c|c|c|}
\hline Lengua & Inglés & Español & Ambas lenguas & Total \\
\hline Número & 71 & 43 & 45 & 159 \\
\hline Porcentaje & $44,70 \%$ & $27 \%$ & $28,30 \%$ & $100 \%$ \\
\hline
\end{tabular}

\subsection{Morfología de los nombres rotuladores}

Los datos sobre las características morfológicas de los nombres rotuladores indican que predominan las nominalizaciones, derivadas principalmente de verbos. Esta tendencia se vincula con la alta densidad léxica de los textos científicos y con el papel de las nominalizaciones como recurso prototípico del discurso científico para la articulación textual. Se ha señalado en la literatura la aparición frecuente y el valor cohesivo de la metáfora gramatical en la lengua científica, principalmente mediante la nominalización para la condensación de la información y la tematización (Halliday, 1993, 1994b, 1998, 2004; Halliday \& Matthiessen, 2014), dos procedimientos que 
distinguen a los RRCC donde aparecen los nombres rotuladores como núcleo nominal.

Si bien los estudios anteriores no se han concentrado en el aspecto morfológico, algunos autores han señalado el predominio de nominalizaciones en diferentes géneros: artículos de investigación en inglés y español (Mur Dueñas, 2003/2004), artículos de semi-divulgación en inglés (Álvarez de Mon y Rego, 2001), artículos de investigación, libros de texto y clases en inglés (Flowerdew \& Forest, 2015), y noticias periodísticas en inglés (Downing, 1997) y español (López Samaniego, 2011, 2013, 2015).

En nuestros datos, la gran mayoría de los nombres nominalizados tienen bases verbales (por ej., assessment, explanation, recommendation, caracterización, observación, razonamiento), tal como ha sido identificado en estudios previos en inglés (Francis, 1986; Ivanic, 1991; Downing, 1997; Schmid, 2000; Mur Dueñas, 2003/2004) y español (Mur Dueñas, 2003/2004; López Samaniego, 2011). Le siguen en número de ocurrencia los nombres rotuladores deadjetivales (por ej., ability, complexity, possibility, capacidad, dificultad, severidad). Por último, identificamos solo algunos nombres derivados de bases nominales (bypothesis/es, methodology, relationship, metodología/s, modalidades, problemática).

Identificamos, también, los nombre compuestos framework y trade-off. Por otra parte, observamos la presencia de nombres simples (por ej., advantage/s, benefits, instances, issue, conceptos, contexto, hecho, idea), también identificados en estudios previos (Álvarez de Mon y Rego, 2001; López Samaniego, 2011). Asimismo, encontramos nombres simples formados por conversión o derivación cero, como approach, view, variables. Como se desprende de los resultados, podemos decir que si bien los nombres simples constituyen un grupo más reducido, también pueden funcionar como nombres rotuladores en los RRCC, dado que tienen el potencial de reconstruir metafóricamente los segmentos textuales (Matthiessen, 1995; Halliday \& Matthiessen, 2014).

\subsection{Semántica de los nombres rotuladores}

\subsubsection{Características generales}

Hemos identificado particularidades semánticas que distinguen los nombres rotuladores de otros nombres comunes y de otras estructuras cohesivas, y los convierten en mecanismos muy productivos para lograr simultáneamente la encapsulación y la rotulación. Se confirmaron tres rasgos básicos que, en mayor o menor grado, caracterizan la semántica de los tipos de nombres que tienen el potencial de funcionar como nombres rotuladores en los RRCC: poseen significados generales, son de carácter inanimado y representan entidades de segundo y tercer orden. Son generales puesto que tienen significados de naturaleza inespecífica; tienen carácter 
inanimado ya que denotan entidades no conscientes y representan entidades de segundo y tercer orden dado que se refieren a eventos, procesos, acontecimientos, proposiciones, nociones y conceptos. De estas tres propiedades, resulta especialmente relevante la noción de generalidad, cualidad fundamental que notablemente distingue la capacidad de un nombre común de funcionar como nombre rotulador para encapsular y etiquetar el contenido de segmentos textuales.

La noción de generalidad (Halliday \& Hasan, 1976; Halliday \& Matthiessen, 2014) constituye una característica sumamente relevante y distintiva de los nombres rotuladores, un aspecto mencionado en la literatura con mucha frecuencia (Ivanic, 1991; Winter, 1992; Francis, 1986, 1994; Schmid, 2000, 2018; Flowerdew, 2003; López Samaniego, 2011, 2015, 2018; Flowerdew \& Forest, 2015; entre otros). Los nombres identificados son de naturaleza inespecífica y poseen un vacío en su estructura semántica (Schmid, 2000) que se completa en cada ocasión de acuerdo con el contenido de los segmentos encapsulados. Este rasgo semántico de inespecificidad los convierte en mecanismos altamente productivos para funcionar como cápsulas conceptuales que adquieren significados específicos en un determinado contexto y solo en relación a la información desplegada en fragmentos del texto. Por ejemplo, unidades como challenge, examples, instances, theory, cambio, factores, patrón y tendencia tienen, según Ivanic (1991), un significado lexicográfico constante al que se puede acceder en un diccionario y un significado particular que se precisa en el contexto específico, cuando el nombre encapsula y rotula el contenido de un segmento del texto. Como se aprecia en el ejemplo (3), el nombre tendencia, cuyo significado lexicográfico es 'propensión o inclinación en los hombres y en las cosas hacia determinados fines' (RAE), adquiere un significado particular en relación con el segmento que encapsula y rotula. Es decir, en el caso que muestra el ejemplo, tendencia se refiere específicamente a la información expresada en el segmento anterior sobre los resultados del estudio reportado en el artículo de investigación.

(3) La menor acumulación en el tratamiento 66\% de raleo puede deberse al menor aporte de material vegetal por parte del estrato arbóreo que, junto al mayor ingreso de energía a la parte inferior del dosel arbóreo modifican las condiciones de sitio para los organismos descomponedores y aceleran la tasa de descomposición (Couteaux, 1995; Pérez Batallón et al., 1998; Borém, 2002; Prescott, 2002; Bosco Imbert, 2004; Muscolo et al., 2007), disminuyendo la posibilidad de acumulación de material sobre el piso forestal en este tratamiento. Esta tendencia se condice con los resultados de otras investigaciones (...)

En este sentido, como propone López Samaniego (2011, 2015), nuestros datos demuestran que debido a la inespecificidad de sus significados los nombres rotuladores tienen la particularidad de denotar un conjunto prácticamente infinito de acciones, conceptos, proposiciones y nociones de diversa naturaleza que pueden agruparse bajo ciertas características en común. Es precisamente por esta razón que estos nombres pueden funcionar como núcleo de los RRCC para englobar y 
caracterizar conceptualmente el contenido de segmentos del texto, un rasgo primordial en el comportamiento discursivo de los RRCC.

Sin embargo, es importante señalar que si bien todos los nombres rotuladores identificados en nuestros datos poseen el rasgo de generalidad, este debe considerarse un rasgo gradual (Halliday \& Hasan, 1976; Winter, 1977, 1992; Schmid, 2000, 2018); por eso es conveniente pensar en términos de un continuum, a lo largo del cual varía el grado de generalidad en los significados de los nombres. Algunos nombres rotuladores presentan inherentemente un alto grado de inespecificidad en sus significados, con escasa carga informativa y conceptual; phenomenon, system, process, situation, acción, caso, hecho, patrón representan nociones muy generales y rotulan una amplia variedad de contenidos proposicionales. Otros nombres, en cambio, denotan significados de naturaleza más específica y poseen ciertos rasgos semánticos que orientan sus significados en ciertos sentidos, como en el caso de challenge, loss, prediction, reduction, ajuste, combinación, eficiencia, evolución. Por esta razón, tienen la capacidad de representar una variedad de información más delimitada y de evocar dimensiones semánticas más restringidas. Así pues, podemos decir que algunos nombres rotuladores pueden abarcar una amplia variedad de procesos, acontecimientos, conceptos, proposiciones y situaciones, mientras que otros nombres pueden completar sus significados con nociones más restringidas. En resumen, si bien todos los nombres rotuladores tienen significados inespecíficos, el grado de inespecificidad varía.

Los conceptos de carácter inanimado (Halliday \& Matthiessen, 2014) y abstracción (Bosque, 1999) también son relevantes para la caracterización semántica de estos nombres. En nuestros datos hemos corroborado que los nombres rotuladores en los RRCC son de carácter inanimado (por ej., approach, complexity, evidence, beneficio, contexto, principio). Asimismo, los podemos definir como abstractos, una característica recurrentemente señalada en la literatura (Francis, 1986; Ivanic, 1991; Hunston \& Francis, 2000; Schmid, 2000; López Samaniego, 2011; Flowerdew, 2003, 2009, 2015; Flowerdew \& Forest, 2015).

El esquema tripartito de clasificación ontológica de Lyons (1977), basado en distintos grados de abstracción, resulta muy apropiado para hacer una caracterización pormenorizada de la naturaleza abstracta de estos nombres (véase también Schmid, 2000; López Samaniego, 2011, 2015). De acuerdo con este esquema, los nombres identificados en nuestro estudio representan entidades de segundo y tercer orden. Los primeros refieren a eventos, procesos y acontecimientos que tienen una ubicación espacio-temporal; en la muestra hemos identificado una importante cantidad de nombres que denotan acciones o eventos, tales como calculation, modification, ajuste, combinación. Por el contrario, los de tercer orden señalan proposiciones (por ej., concerns, expectations, prediction, dilema, necesidad, premisa) y nociones conceptuales (por ej., knowledge, 'razón'). Como señala Lyons, a diferencia de los nombres de segundo 
orden, que se caracterizan por ser o no ser reales, los nombres de tercer orden se caracterizan por tener valor de verdad y por la posibilidad de ser afirmados o negados. En síntesis, los nombres rotuladores de segundo orden son más abstractos que los nombres que designan personas y objetos físicos dado que no tienen existencia estable en la dimensión del tiempo, pero menos abstractos que los nombres que señalan proposiciones, conceptos e ideas porque ocurren en el mundo físico. Por último, entre los nombres identificados encontramos tres, 'párrafo', 'punto' y 'sección', de orden metalingüístico que mencionan partes o componentes del texto mismo, los cuales no están contemplados en el esquema de Lyons.

\subsubsection{Categorización semántica: Significados de los nombres rotuladores}

En esta sección nos centramos en el análisis de los significados de los nombres. Cabe hacer una puntualización respecto de la categorización que proponemos en nuestro estudio: como era de prever, en el proceso de clasificación nos enfrentamos a dificultades para establecer categorías semánticas mutuamente excluyentes, que puedan delimitarse de manera precisa. Esta dificultad para sistematizar nombres rotuladores en categorías discretas es señalada en la literatura (Francis, 1986, 1994; Ivanic, 1991; Schmid, 2000; Flowerdew \& Forest, 2015). En respuesta a esta dificultad, algunos autores han apelado a la semántica de prototipos (por ej., Kleiber 1995; Cruse, 2006) y han definido las categorías a base de un nombre que funciona como prototipo o núcleo central, que unifica la categoría, y nombres menos prototípicos que se aproximan más o menos al prototipo. Desde esta perspectiva, algunos nombres representan ejemplos característicos de una categoría, mientras que otros representan casos más periféricos; del mismo modo hemos procedido en nuestro estudio, partiendo de la convicción de que los límites de las categorías conceptuales no son rígidos y estrictos sino que pueden ser imprecisos.

Varias categorizaciones presentes en estudios anteriores sirvieron de base a nuestra sistematización: 'nombres lingüísticos' (Schmid, 2000, 2018), 'de actividades verbales' (Francis, 1986, 1994) o 'de locución' (Francis, 1994; Flowerdew \& Forest, 2015), 'textuales' (Francis, 1986, 1994), 'de hechos' (Flowerdew \& Forest, 2015) o 'eventivos' (Schmid, 2000, 2018), 'de cognición' (Francis, 1986), 'de procesos mentales’ (Francis, 1994; Schmid, 2000, 2018) o 'ideas' (Flowerdew \& Forest, 2015), 'modales' (Schmid, 2000, 2018; Flowerdew \& Forest, 2015) y 'circunstanciales' (Schmid, 2000, 2018; Flowerdew \& Forest, 2015). Nuestra categorización también se sustenta en la literatura gramatical (Bosque, 1999; Lacuesta \& Bustos Gisbert, 1999; Picallo, 1999; RAE, 2009).

Debido a la especificidad del artículo de investigación, y a partir de los resultados que emergieron en nuestro estudio, para ordenar y categorizar el universo de los nombres rotuladores de nuestros datos fue necesario realizar una revisión y una reelaboración teórica de las categorías existentes en la literatura e incluso proponer 
nuevas categorías que no han sido contempladas en estudios previos, a saber: nombres 'de causa y finalidad', 'de investigación', 'de propiedades, cualidades y estados' y 'clasificativos'. En la Tabla 2 se presenta la sistematización que proponemos y algunos ejemplos a modo de ilustración. Los nombres en negrita representan los miembros prototípicos de las categorías, ordenadas conceptualmente en dos clases principales: las de orden metadiscursivo, categorías 1 y 2, y las que señalan distintas actividades propias al proceso de investigación, categorías 3-10.

Tabla 2. Esquema de categorización semántica de los nombres rotuladores presentes en los rótulos cohesivos identificados en artículos de investigación de las ciencias agrarias.

\begin{tabular}{|c|c|c|c|}
\hline & Categoría & Ejemplos en español & Ejemplos en inglés \\
\hline \multirow[t]{2}{*}{ Metadiscursivo } & 1) Lingüísticos/comunicativos & $\begin{array}{l}\text { comentario, cita, } \\
\text { conclusiones, } \\
\text { explicación }\end{array}$ & $\begin{array}{l}\text { conclusions, } \\
\text { explanation, examples }\end{array}$ \\
\hline & 2) Textuales & punto & paragraph, sections \\
\hline \multirow{8}{*}{$\begin{array}{l}\text { Proceso de } \\
\text { investigación }\end{array}$} & 3) Hechos, eventos y procesos & $\begin{array}{l}\text { hecho, acción, } \\
\text { cambio, evolución, } \\
\text { proceso }\end{array}$ & $\begin{array}{l}\text { process, } \\
\text { phenomenon, } \\
\text { changes, development }\end{array}$ \\
\hline & 4) Causa y finalidad & $\begin{array}{l}\text { causa, efecto, fin, } \\
\text { motivo, objetivos }\end{array}$ & $\begin{array}{l}\text { effect, end, reasons, } \\
\text { purposes, goal }\end{array}$ \\
\hline & 5) Propiedades, cualidades y estados & $\begin{array}{l}\text { características, } \\
\text { propiedades, estado }\end{array}$ & $\begin{array}{l}\text { characteristics, } \\
\text { aspects, attributes, } \\
\text { ability }\end{array}$ \\
\hline & 6) Mentales/cognitivos & $\begin{array}{l}\text { razonamiento, } \\
\text { análisis, } \\
\text { caracterización }\end{array}$ & $\begin{array}{l}\text { concepts, estimate, } \\
\text { rationale, theory }\end{array}$ \\
\hline & 7) Evaluativos y epistémicos & $\begin{array}{l}\text { beneficio, tendencia, } \\
\text { dilema, dificultad }\end{array}$ & $\begin{array}{l}\text { benefits, possibility, } \\
\text { trend, challenge }\end{array}$ \\
\hline & 8) Investigación & $\begin{array}{l}\text { método, datos, } \\
\text { esquema, criterios }\end{array}$ & $\begin{array}{l}\text { data, model, } \\
\text { technique, system, } \\
\text { procedure }\end{array}$ \\
\hline & 9) Clasificativos & grupos, tipos & classes, types \\
\hline & 10) Circunstancias & $\begin{array}{l}\text { contexto, situación, } \\
\text { modalidades }\end{array}$ & $\begin{array}{l}\text { situation, scenario, } \\
\text { instances }\end{array}$ \\
\hline
\end{tabular}

El grupo 1, 'lingüísticos/comunicativos', incluye nombres metalingüísticos que designan específicamente actividades verbales o actos de comunicación (por ej., prediction, comentario). De hecho, muchos de los nombres identificados representan nominalizaciones de los llamados verbos de lengua que denotan actividades de habla (RAE, 2009), como por ejemplo questions y explicación. Los nombres de la categoría 1 también pueden interpretarse como los resultados de actividades lingüísticas, por ejemplo en el caso de information y cita. En cualquier interpretación, los nombres se emplean para rotular algo que se ha escrito en el texto o algo que se ha sido dicho y sobre lo cual se está informando. 
$\mathrm{Al}$ igual que el grupo 1, el grupo 2, 'textuales', representa nombres de naturaleza metalingüística, que se refieren específicamente a la estructura o elementos particulares del discurso, tales como partes del texto (por ej., paragraph, section).

El grupo 3, 'hechos, eventos y procesos', comprende nombres de acción con interpretación eventiva que designan una variedad de actividades y acontecimientos (por ej., modification, comportamiento) o nombres de resultado con interpretación resultativa que designan los efectos producidos por dichas acciones y procesos (por ej., loss, distribución) (Bosque, 1999; Picallo, 1999; RAE, 2009). En este sentido, algunos nombres pueden interpretarse como actos y procesos o como el efecto o producto de un proceso (por ej., increase, reduction, ajuste, cambio). El contenido de acción de los nombres del grupo 3 se entiende en un sentido amplio que incluye no solo acciones, actos y actividades que se llevan a cabo intencionalmente con un objetivo, sino también fenómenos, acontecimientos, eventos y procesos que no se atribuyen a agentes (Lyons, 1977). En nuestros datos, las acciones producidas intencionalmente corresponden en general a aquellas que los investigadores llevaron a cabo en el proceso de investigación y que están siendo reportadas en los artículos. Son actividades en las que los escritores estuvieron involucrados (por ej., adjustments, refinement, práctica, cambio). Los eventos y procesos que no se atribuyen a agentes corresponden a sucesos o fenómenos que también toman lugar en el mundo físico pero sin la participación de los investigadores (por ej., behaviour, development, comportamiento, evolución). También se pueden diferenciar nombres que provienen de verbos dinámicos (por ej., development, evolución), que predominan en nuestros datos, y aquellos que provienen de verbos estáticos que señalan estados o relaciones entre entidades (por ej., relación).

En términos generales, podemos decir que las categorías 1 y 3 se corresponden con los nombres de acción y efecto, de evento o suceso, y nombres eventivos discutidos en las gramáticas (Lacuesta \& Bustos Gisbert, 1999; Picallo, 1999; RAE, 2009; Varela Ortega \& Piera, 1999). Se trata de nombres que designan acciones o sucesos, algo que acontece o que se sitúa en un espacio de tiempo y tienen límites temporales (Bosque, 1999; Picallo, 1999). Como ya hemos señalado, este grupo de nombres puede tener una doble interpretación, ya sea la acción o suceso o el efecto del evento (Bosque, 1999; Picallo, 1999; RAE, 2009).

El grupo 4, 'causa y finalidad', incluye nombres que se utilizan para indicar motivos e intenciones u objetivos y propósitos, como en el caso de end, purposes, reasons, causa, efecto y propósito.

El grupo 5, 'propiedades, cualidades y estados', contiene nombres que señalan características intrínsecas o cualidades atribuidas a diferentes tipos de entidades y actividades (por ej., ability, attributes, capacidad, propiedades), como así también estados (por ej., condition, estado). Estos nombres se asocian con los nombres de cualidad, 
estado y condición (Lacuesta \& Bustos Gisbert, 1999) o los nombres de cualidad formados a partir de adjetivos que designan propiedades (RAE, 2009). Incluyen no solo nominalizaciones de adjetivos que denotan propiedades o cualidades particulares (por ej., ability, diversidad) sino también nombres que engloban y designan distintas características (por ej., characteristics, atributos).

El grupo 6, 'mentales/cognitivos', incluye nombres que describen ideas, estados mentales, operaciones o procesos cognitivos atribuidos a la mente o los productos resultantes de los mismos. En líneas generales, estos nombres se relacionan con los nombres asociados a verbos de afección que denotan estados psicológicos, conocimiento, percepción y diversos estados de conciencia (Picallo, 1999; RAE, 2009). Se asocian también a los procesos mentales que denotan percepción y cognición (Halliday, 1994a). Incluyen nominalizaciones que representan procesos mentales y cognitivos (por ej., association, comparison, estimación, razonamiento) y nombres que no derivan de verbos y denotan el resultado del procesamiento de operaciones cognitivas y pensamientos (por ej., concepts, rationale, theory, concepto, premisa). Por otra parte, al igual que ciertos nombres del grupo 3 ('hechos, eventos y procesos'), algunos nombres del grupo 6 tienen una doble interpretación, ya que pueden referirse tanto al resultado como al proceso (por ej., estimate, análisis).

En el grupo 7, 'evaluativos/epistémicos', se incluyen nombres que denotan la actitud y evaluación explícita del escritor (por ej., advantage, problem, dilema, progreso) y nombres que se emplean para atenuar el grado de certeza acerca de las afirmaciones sobre las observaciones y los resultados reportados (por ej., possibility, tendencia). En general, estos nombres se relacionan con los nombres asociados a verbos de afección que denotan impresiones, emociones y actitudes (Picallo, 1999; RAE, 2009) у a procesos mentales que denotan afección (Halliday, 1994a). En términos de la gramática sistémica funcional, los nombres del grupo 7 conllevan significado interpersonal. Los evaluativos permiten expresar valoraciones sobre teorías, técnicas, materiales e instrumentos, situaciones, resultados y proposiciones propias o de otros. Los epistémicos permiten señalar el compromiso y posicionamiento de los autores al mitigar el nivel de certeza y veracidad de las proposiciones (Lyons, 1977). Mientras que los nombres evaluativos se definen en relación a la oposición positivo-negativo, los epistémicos se definen sobre la oposición certeza-incertidumbre.

La categoría 8, 'investigación', representa nombres que denotan conceptos, nociones y expresiones relacionadas con el proceso de investigación (por ej., approach, mecanismo). Si bien algunos de estos nombres pueden asociarse a otras categorías, como por ejemplo 'hechos, eventos y procesos', hemos individualizado el grupo 8 dado que incluye nombres con significados estrechamente relacionados con el propósito comunicativo del artículo de investigación. La mayoría de estos nombres representan aspectos metodológicos, como methodology, procedure, technique esquema, método, protocolo. Otros se relacionan con la presentación de resultados, como 
evidence, findings, datos y resultados. También se incluyen en este grupo nombres que hacen referencia a otros estudios, como literature y trabajos. El grupo también contempla una serie de nombres empleados para referirse a normas y criterios que regulan las actividades y procedimientos en el proceso de investigación u otras actividades que se ejecutan en el mundo de la experiencia, por ejemplo parameters, rules, criterios y principios.

La categoría 9, 'clasificativos', incluye nombres de clase utilizados para categorizar o agrupar conceptos y entidades (RAE, 2009), como classes y tipo. Estos nombres representan generalmente los denominados species nouns en inglés (por ej., kind) (Biber et al., 1999). También hemos incluido el nombre cuantificativo grupo (Bosque, 1999; RAE, 2009). Cabe aclarar que los nombres clasificativos también podrían asociarse al grupo 8, 'de investigación', dado que, al igual que los nombres como parameters y criterios, se utilizan para agrupar y sistematizar. No obstante, hemos decidido apartarlos puesto que aparecen generalmente en estructuras pseudopartitivas que ejercen la función de agrupar entidades e introducen sintagmas nominales sin determinante (Biber et al., 1999; RAE, 2009).

Por último, pertenecen a la categoría 10, 'circunstancias', nombres utilizados para referirse y englobar lo que acontece en el marco de un hecho, acción o suceso y las circunstancias en las que estos suceden, encuadrándolos y delimitándolos en un contexto al presentar información, por ejemplo, sobre dónde y cuándo se manifiesta un acontecimiento, como en el caso de phase, scenario, contexto y situación. El grupo también incluye nombres que se utilizan para referirse a propiedades aspectuales de las acciones y los acontecimientos al sintetizar la forma en que algo se lleva a cabo o algo sucede, como modo ${ }^{4}$.

Como se advierte en las categorías que proponemos, existe una amplia variedad de nombres con distintos significados. Los nombres más recurrentes en los datos analizados en inglés son result/s, difference/s, finding/s, factors, bypothesis/es, observation/s, reason $/ s$, section $/ s$, effect/s, approach, processes, condition/s. Por otra parte, los que más se repiten en español son resultado/s, efecto/s, factor/es, caso/s, situación/es, condición/es, objetivo/s, diferencia/s, metodología/s, dato/s. Teniendo en cuenta que estos nombres aparecen regularmente en los rótulos cohesivos identificados, se puede inferir que, en general, se usan para encapsular y rotular información acerca de los resultados de las investigaciones reportadas en los artículos. Más allá de la frecuencia de aparición de los nombres y haciendo abstracción de posibles diferencias genéricas y disciplinares, nuestros resultados coinciden con las tendencias generales de investigaciones previas, dado que varios de los nombres rotuladores identificados en nuestro estudio han sido señalados en otros trabajos en inglés (Francis 1986; Hunston \& Francis, 2000; Hinkel, 2004; Gray, 2010; Flowerdew \& Forest, 2015) y en español (Álvarez de Mon y Rego, 1999; López Samaniego, 2011). 
Independientemente del uso preferencial de ciertos nombres rotuladores, las diez categorías propuestas en el presente estudio ponen de relieve la variedad de significados de los nombres que aparecen como núcleo nominal de los rótulos cohesivos empleados en los artículos de investigación de las ciencias agrarias que conforman la muestra. Las nociones de diversa naturaleza que designan los nombres pueden agruparse en dos clases principales: el contexto discursivo que refleja el proceso de comunicación científica ('lingüísticos/ comunicativos', 'textuales') y el mundo extralingüístico que refleja la actividad de investigación ('hechos, eventos y procesos', 'propiedades, cualidades y estados', 'mentales/cognitivos', 'circunstancias', 'causa y finalidad', 'evaluativos y epistémicos', 'investigación' y 'clasificativos'). La mayoría de los nombres rotuladores denotan nociones asociadas al proceso de investigación, principalmente en las categorías 'investigación', 'evaluativos y epistémicos', 'hechos, eventos y procesos' y 'mentales/cognitivos'.

La diversidad de significados que se refleja en la clasificación conceptual de los nombres rotuladores permite destacar dos aspectos: por un lado, la amplia variedad de opciones disponibles para conceptualizar y etiquetar fragmentos textuales en los artículos de investigación de las ciencias agrarias, lo cual confirma el potencial de los nombres rotuladores para realizar simultáneamente los procedimientos de encapsulación y rotulación. Por otro lado, puede constatarse que, en los artículos de investigación analizados, los nombres se emplean para rotular actos lingüísticos, eventos, acciones, proposiciones, conceptos, ideas, situaciones y nociones asociadas al proceso de investigación.

Como ya dijimos, la mayoría de los nombres rotuladores denotan nociones asociadas al proceso de investigación. Este resultado nos permite constatar coincidencias con los datos aportados en estudios previos acerca del uso de nombres rotuladores en artículos de investigación de diferentes disciplinas, los cuales han identificado en inglés (Charles, 2003) y en inglés y español (Mur Dueñas, 2003/2004) el predominio de nombres no metalingüísticos (por ej., efecto, resultado, investigación) en oposición al escaso uso de nombres metalingüísticos (por ej., punto, distinción, expresión). Del mismo modo, nuestros resultados corroboran la alta frecuencia de nombres relacionados al proceso de investigación identificada en estudios previos: nombres empleados para presentar y discutir resultados (por ej., difference, effect, finding, results) (Swales, 2005; Aktas \& Cortés, 2008; Gray, 2010; Gray \& Cortés, 2011; Mousavi \& Rauof Moini, 2014; Flowerdew \& Forest, 2015), nombres de procesos o resultados mentales (por ej., hypothesis, understanding) (Gray, 2010) y nombres que indican la metodología (por ej., approach, method/s, technique) (Swales, 2005; Gray \& Cortes, 2011; Flowerdew \& Forest, 2015). Sin embargo, nuestros resultados difieren de otras investigaciones que detectaron el uso frecuente de nombres rotuladores metadiscursivos para señalar partes del texto (por ej., article, paper, section, figure, study) (Moreno, 2004; Swales, 2005; Gray \& Cortes, 2011). Posiblemente 
las diferencias se deban a particularidades de las disciplinas estudiadas, dado que ninguno de los estudios mencionados se ha enfocado en las ciencias agrarias.

\subsection{Pragmática de los nombres rotuladores}

Como hemos demostrado, todos los nombres rotuladores tienen la propiedad de encapsular y etiquetar fragmentos textuales; la rotulación depende de las variadas alternativas de acuerdo con los significados de los nombres y el contexto de empleo específico. La pregunta que abordamos en esta sección se vincula con la dimensión pragmática de estos nombres, es decir, cuál función cumplen en el entramado textual que crean. Sobre la base de nuestros datos, ha sido posible identificar dos funciones: por un lado, señalan significados interpersonales, más orientados hacia el escritor, para la expresión de actitud y valoración en la clasificación conceptual de los segmentos encapsulados (4). Por otro lado, asumen significados textuales, más orientados hacia el texto mismo, para señalar metadiscursivamente la organización de la información (5):

(4) However, the labeling of sparingly soluble P sources by neutron irradiation is relatively costly and can sometimes lead to the labeling of accompanied cations (Armstrong et al. 1993). A 32 $\mathrm{P}$ dilution technique which involves labeling the bio-available soil $\mathrm{P}$ instead of $\mathrm{P}$ sources overcomes this problem (Zapata \& Axmann 1995).

(5) Harper (1967) sostiene que el esfuerzo reproductivo de una maleza está inversamente relacionado con el grado de madurez del hábitat que explora. En el caso de C. erecta, la estrategia colonizadora (semillas) particionó más a semillas que la estrategia asociada con hábitats más maduros (rizomas). Otra explicación es que la diferente partición de recursos puede estar relacionada con las variaciones en el peso de las plantas de acuerdo a su origen, el cual fue mayor en las provenientes de rizomas.

Puede concluirse que los nombres rotuladores constituyen piezas fundamentales en los RRCC, pues permiten señalizar evaluación y organización del texto, como ha sido señalado en la literatura (Halliday \& Hasan, 1976; Francis, 1986, 1994; Winter, 1992; Schmid, 2000; Flowerdew, 2003, 2015; López Samaniego, 2011; Flowerdew \& Forest, 2015; Borreguero Zuloaga, 2018; entre otros).

La carga valorativa de los nombres permite expresar actitud y evaluación positiva o negativa sobre la información, o probabilidad y grados de certeza sobre la veracidad de las proposiciones. En este sentido, son más productivos en su función evaluativa los nombres que tienen un componente valorativo intrínseco (por ej., advantage, benefits, limitations, possibility, uncertainties, dilema, discrepancia, necesidad, problemática, tendencia), en oposición a los más neutros (por ej., concepts, factors, operation, procedure, purposes, acción, causa, esquema, patrón, práctica). En cualquier caso, dado su contenido léxico, el nombre rotulador permite categorizar conceptualmente e indicar cómo un determinado fragmento debe interpretarse, pues la selección del nombre es un proceso que supone remitir al segmento encapsulado desde una perspectiva. Es decir, el grado de valoración puede ser más o menos visible, pero estaría siempre 
presente, puesto que el proceso de rotulación implicaría una toma de posición por parte del escritor respecto del contenido del texto.

Es decir, si bien el grado de significado interpersonal de los nombres varía, todos construyen significados interpersonales, puesto que clasifican los segmentos encapsulados a partir del posicionamiento de los escritores (Francis, 1986, 1994; Schmid, 2000; Charles, 2003; López Samaniego, 2013, 2015; Flowerdew \& Forest, 2015). Excepto en el caso de los nombres textuales, que remiten a partes del texto, la selección de los nombres es pragmática y en ocasiones podría ser estratégica, ya que el escritor puede intencionalmente acercarse o distanciarse del texto (Francis, 1994).

Lo expuesto hasta aquí permite plantear que algunos nombres rotuladores son más neutros mientras que otros son inherentemente actitudinales en sus significados, como se ha observado en estudios previos (Francis, 1986, 1994; Schmid, 2000; López Samaniego, 2011). En el primer grupo se encuentran los nombres cuyos significados no connotan sentidos positivos o negativos (por ej., description, relación). El segundo grupo constituye nombres evaluativos que, en virtud de sus significados conceptuales valorativos, permiten la expresión explícita de actitud y valoración, ya sea positivamente (por ej., advantage, beneficio) o negativamente (por ej., complexity, problemática), y nombres epistémicos que permiten expresar el grado de compromiso con la información (por ej., possibility, tendencia).

En consecuencia, proponemos disponer los nombres rotuladores en un continuum que se desplaza desde un extremo con los nombres más neutros a otro extremo con los nombres con mayor carga evaluativa, los cuales reflejan de manera más explícita la actitud del escritor. En coincidencia con Francis (1986, 1994), consideramos que en el extremo de neutralidad se encuentran los nombres textuales, como paragraph, que indican propiedades formales del texto, mientras que en el extremo opuesto se encuentran los nombres evaluativos y epistémicos, que señalan valoración y grados de certeza, como dilema y uncertainties.

Por otra parte, hemos observado que los nombres rotuladores que integran los rótulos cohesivos también desempeñan el papel de recursos metadiscursivos, una función señalada en la literatura previa (por ej., Francis, 1986, 1994; Winter, 1992; López Samaniego, 2011; Flowerdew \& Forest, 2015). Los estudios previos han asumido dos posiciones: los que consideran que solo un subgrupo de nombres son metatextuales y los que postulan que todos los nombres rotuladores se emplean como recursos metadiscursivos. Consideramos que ambas posiciones encuentran fundamento en nuestro estudio.

Respecto de la primera posición, y asumiendo una definición de metadiscurso o metatexto restringida a la función mediante la cual se comenta explícitamente sobre la estructura del texto (Ädel, 2006; Fandrych \& Graefen, 2002), podemos establecer que son elementos metatextuales prototípicos los nombres rotuladores que explícitamente 
permiten guiar al lector y dirigir su atención hacia la organización textual (Fandrych \& Graefen, 2002). Como ya hemos argumentado, en nuestra sistematización son de naturaleza metadiscursiva los nombres 'lingüísticos/comunicativos' (por ej., questions, explicación) y los 'textuales' (por ej., paragraph, punto). En este sentido, coincidimos con Francis $(1986,1994)$, Charles (2003) y Borreguero Zuloaga (2018).

Por otra parte, parece acertado sostener también la segunda posición, considerando que los nombres rotuladores que aparecen en los RRCC tienen la propiedad de la reflexividad y, por ende, categorizan conceptualmente información textual (no la realidad extralingüística). Si bien es cierto que algunos nombres representan entidades de segundo orden, como eventos y acciones, la función característica de los nombres rotuladores -indicar cómo deber interpretarse un segmento textual, establecer conexión y organizar la estructura del texto- está muy próxima al funcionamiento metadiscursivo. En este sentido, coincidimos con Flowerdew (2015), Flowerdew y Forest (2015) y Winter (1992) en considerar que todos los nombres rotuladores son recursos metalingüísticos, dado que señalan y designan partes del texto, permiten organizarlos y sustituyen a otros elementos textuales.

\section{CONCLUSIONES}

En este trabajo hemos elaborado un inventario de los nombres rotuladores presentes en la muestra de artículos de ciencias agrarias en inglés y español, y hemos aportado datos descriptivos sobre sus características lingüísticas, información que nos permite explicar la naturaleza de estos nombres, siendo algunos más prototípicos que otros, y su capacidad de funcionar como componentes centrales de los rótulos cohesivos.

En primer lugar, desde el punto de vista formal, podemos afirmar que en el conjunto de nombres rotuladores que relevamos predominan las nominalizaciones deverbales, un rasgo prototípico del discurso científico (Halliday, 1993, 1998, 2004). Cabe señalar que la morfología de los nombres rotuladores constituye un aspecto poco explorado sistemáticamente en la literatura, por lo que nuestros resultados aportan evidencia novedosa sobre las propiedades formales de los nombres que pueden funcionar como nombres rotuladores.

En segundo lugar, desde el punto de vista semántico, se destacan tres rasgos centrales que distinguen a los nombres rotuladores presentes en los RRCC y los hacen potencialmente productivos para funcionar como mecanismos de encapsulación y rotulación conceptual: poseen significados generales, son de carácter inanimado y representan entidades de segundo y tercer orden. Las categorías semánticas que hemos propuesto reflejan una amplia diversidad de significados para conceptualizar la información textual. 
Por último, si bien todos los nombres rotuladores permiten la reconstrucción conceptual de los fragmentos encapsulados, algunos señalan explícitamente significados interpersonales que reflejan la actitud y valoración del escritor, mientras que otros poseen inherentemente significados textuales que contribuyen a la organización de los textos. El grado de interpretación y conceptualización dependerá del valor actitudinal o epistémico de los nombres. Cualquiera sea el caso, dada la diversidad de significados que hemos identificado en nuestro estudio, podemos asumir que se dispone de un amplio repertorio de opciones para encapsular y caracterizar conceptualmente fragmentos textuales en los artículos de investigación de las ciencias agrarias. Desde un punto de vista discursivo, las características pragmáticas se tornan importantes para el comportamiento de los RRCC, cuya función está estrechamente relacionada con la evaluación y estructuración de los textos.

Para concluir, es sabido que los nombres rotuladores se definen como una clase funcional (Schmid, 2000, 2018; López Samaniego, 2011, 2015; Flowerdew \& Forest, 2015). En este estudio se han hecho visibles las propiedades morfológicas, semánticas y pragmáticas que definen la naturaleza de estos nombres y que los hacen productivos para funcionar como componentes centrales de los RRCC para encapsular, clasificar conceptualmente y establecer enlaces entre fragmentos textuales.

Nuestros resultados surgen de una muestra representativa de un género y una disciplina científica. Advertimos coincidencias con estudios previos acerca del uso de nombres rotuladores en artículos de investigación de diferentes disciplinas, por ejemplo la alta frecuencia de nombres relacionados al proceso de investigación, tendencia que se atribuye, por un lado, al propósito retórico del artículo de investigación de construir conocimiento científico y, por otro lado, a la naturaleza semántica de los nombres rotuladores, que los hace productivos y frecuentes en diferentes textos académicos y disciplinas. No obstante, es necesario relativizar las generalizaciones y contar con evidencia empírica en otras áreas, una línea de investigación que ya ha comenzado con estudios que demuestran variación en el uso de nombres rotuladores debido a la especificidad y las particularidades de los géneros y las disciplinas (Schmid, 2000; Charles, 2003; Flowerdew, 2015; Flowerdew \& Forest, 2015) y la familiaridad de los escritores con un campo disciplinar específico (Charles, 2003; Flowerdew \& Forest, 2015). Para continuar con esta línea investigativa, futuros estudios podrían explorar las propiedades de los nombres rotuladores presentes en los rótulos cohesivos en artículos de investigación y géneros de otros campos disciplinares y determinar el posible grado de variación o especificad del inventario de nombres rotuladores propuestos en nuestro estudio. Estudios de este tipo permitirían avanzar en la caracterización de los nombres rotuladores y en el estudio del procedimiento discursivo que se logra a partir del uso de los rótulos cohesivos en el discurso de la ciencia. 


\section{REFERENCIAS BIBLIOGRÁFICAS}

Ädel, A. (2006). Metadiscourse in L1 and L2 English. Amsterdam: John Benjamins.

Álvarez de Mon y Rego, I. (1999). La cohesión del texto científico-técnico. Un estudio contrastivo inglés-español. Tesis doctoral no publicada, Universidad Complutense de Madrid, Madrid, España.

Álvarez de Mon y Rego, I. (2001). Encapsulation and prospection in written scientific English. Estudios Ingleses de la Universidad Complutense, 9, 81-101.

Aktas, R. \& Cortés, V. (2008). Shell nouns as cohesive devices in published and ESL student writing. Journal of English for Academic Purposes, 7(1), 3-14.

Benítez Castro, M. A. (2013). Formal, syntactic, semantic and textual features of English shell nouns. Tesis doctoral, Universidad de Granada, Granada, España.

Benítez Castro, M. A. \& Thompson, P. (2015). Shell-nounhood in academic discourse. A critical state-of-the art review. International Journal of Corpus Linguistics, 20(3), 378-404.

Biber, D. (2008). Representativeness in corpus design. En T. Fontenelle (Ed.), Practical lexicography. A reader (pp. 63-87). Oxford: Oxford University Press.

Biber, D., Johansson, S., Leech, G., Conrad, S. \& Finegan, E. (1999). Longman grammar of spoken and written English. Essex: Pearson Education.

Borreguero Zuloaga, M. (2018). Los encapsuladores anafóricos: Una propuesta de clasificación. Caplletra, 64, 179-203.

Bosque, I. (1999). El nombre común. En I. Bosque \& V. Demonte (Eds.), Gramática descriptiva de la lengua española. Sintaxis básica de las clases de palabras Vol. 1 (pp. 375). Madrid: Espasa Calpe.

Charles, M. (2003). 'This mystery...': A corpus-based study of the use of nouns to construct stance in theses from two contrasting disciplines. Journal of English for Academic Purposes, 2(4), 313-326.

Connor, U. \& Moreno, A. (2005). Tertium comparationis: A vital component in contrastive rhetoric. En P. Bruthiaux, D. Atkinson, W. Eggington, W. Grabe \& V. Ramanathan (Eds.), Directions in applied linguistics: Essays in honour of Robert B. Kaplan (pp.153-164). Clevedon: Multilingual Matters.

Crismore, A. \& Farnsworth, R. (1990). Metadiscourse in popular and professional science discourse. En W. Nash (Ed.), The writing scholar. Studies in academic discourse (pp. 118-136). Newbury Park: Sage. 
Cruse, A. (1986). Lexical semantics. Cambridge: Cambridge University Press.

Cruse, A. (2000). Meaning in language: An introduction to semantics and pragmatics. Oxford: Oxford University Press.

Cruse, A. (2006). A glossary of semantics and pragmatics. Edinburgh: Edinburgh University Press

Downing, A. (1997). Encapsulating discourse topics. Estudios Ingleses de la Universidad Complutense, 5, 147-168.

Fandrych, C. \& Graefen, G. (2002). Text commenting devices in German and English academic articles. Multilingua, 21, 17-43.

Flowerdew, J. (2003). Signalling nouns in discourse. English for Specific Purposes, 22(4), $329-346$.

Flowerdew, J. (2009). Use of signalling nouns in a learner corpus. En J. Flowerdew \& M. Mahlberg (Eds.), Lexical cohesion and corpus linguistics (pp. 85-102). Ámsterdam: John Benjamins.

Flowerdew, J. (2015). Revisiting metadiscourse: Conceptual and methodological issues concerning signalling nouns. Ibérica, 29, 15-34.

Flowerdew, J. \& Forest, R. W. (2015). Signalling nouns in English. A corpus-based discourse approach. Cambridge: Cambridge University Press.

Francis, G. (1986). Anaphoric nouns. Discourse analysis monograph no. 11. Birmingham: English Language Research.

Francis, G. (1994). Labelling discourse: An aspect of nominal-group lexical cohesion. En M. Coulthard (Ed.), Advances in written text analysis (pp. 179-190). Londres: Routledge.

Gray, B. (2010). On the use of demonstrative pronouns and determiners as cohesive devices: A focus on sentence-initial this/that in academic prose. Journal of English for Academic Purposes, 9(3), 167-183.

Gray, B. \& Cortés, V. (2011). Perception vs. evidence: An analysis of this and these in academic prose. English for Specific Purposes, 30(1), 31-43.

Halliday, M.A.K. (1993). On the language of physical science. En M.A.K. Halliday (Ed.), Writing science: Literacy as discursive power (pp. 59-75). Londres: The Falmer Press.

Halliday, M.A.K. (1994a). An introduction to functional grammar. Londres: The Falmer Press. 
Halliday, M.A.K. (1994b). The construction of knowledge and value in the grammar of scientific discourse, with reference to Charles Darwin's The Origin of species. En M. Coulthard (Ed.), Advances in written text analysis (pp. 136-156). Londres: Routledge.

Halliday, M.A.K. (1998). Things and relations. Regrammaticising experience as technical knowledge. En J. R. Martin \& R. Veel (Eds.), Reading science. Critical and functional perspectives on discourses of science (pp. 185-236). Londres: Routledge.

Halliday, M.A.K. (2004). The language of science. Londres: Continuum.

Halliday, M.A.K. \& Hasan, R. (1976). Cohesion in English. Londres: Longman.

Halliday, M.A.K. \& Matthiessen, C. M. I. M. (2014). Halliday's introduction to functional grammar. Londres: Routledge.

Hinkel, E. (2004). Teaching academic ESL writing. Practical techniques in vocabulary and grammar. Mahwah: Lawrence Erlbaum.

Huddleston, R. (1984). Introduction to the grammar of English. Cambridge: Cambridge University Press.

Hunston, S. (2002). Corpora in applied linguistics. Cambridge: Cambridge University Press.

Hunston, S. \& Francis, G. (2000). Pattern grammar. A corpus-driven approach to the lexical grammar of English. Amsterdam: John Benjamins.

Ivanic, R. (1991). Nouns in search of a context: a study of nouns with both open- and closed-system characteristics. IRAL International Review of Applied Linguistics in Language Teaching, 29(2), 93-114.

Jiang, F. \& Hyland, K. (2016). Nouns and academic interactions: A neglected feature of metadiscourse. Applied Linguistics, 37, 1-25.

Jiang, F. \& Hyland, K. (2017). Metadiscursive nouns: Interaction and cohesion in abstract moves. English for Specific Purposes, 46, 1-14.

Kleiber, G. (1995). La semántica de los prototipos. Categoría y sentido léxico. Madrid: Visor.

Lacuesta, R. \& Bustos Gisbert, E. (1999). La derivación nominal. En I. Bosque \& V. Demonte (Eds.), Gramática descriptiva de la lengua española. Entre la oración y el discurso. Morfología Vol. 3 (pp. 4505-4594). Madrid: Espasa Calpe.

López Samaniego, A. (2011). La categorización de entidades del discurso en la escritura profesional. Las etiquetas discursivas como mecanismo de cohesión léxica. Tesis doctoral, Universitat de Barcelona, Barcelona, España. 
López Samaniego, A. (2013). Las etiquetas discursivas: Mantenimiento a la construcción del referente. ELUA, 27, 167-197.

López Samaniego, A. (2015). Etiquetas discursivas, hiperónimos y encapsuladores: Una propuesta de clasificación de las relaciones de cohesión referencial. Revista de Filología Hispánica, 31(2), 435-462.

López Samaniego, A. (2018). La encapsulación nominal en el discurso académicocientífico oral y escrito: Patrones de aparición. Caplletra, 64, 129-152.

Lyons, J. (1977). Semantics (Vols. 1-2). Cambridge: Cambridge University Press.

Mahlberg, M. (2005). English general nouns. A corpus theoretical approach. Ámsterdam: John Benjamins.

Matthiessen, C. M. I. M. (1995). Lexicogrammatical cartography: English systems. Tokyo: International Language Sciences.

Moreno, A. (2004). Retrospective labeling in premise-conclusion metatext: An English-Spanish contrastive study of research articles on business and economics. Journal of English for Academic Purposes, 3(4), 321-339.

Moreno, A. (2008). The importance of comparable corpora in cross-cultural studies. En U. Connor, E. Nagelhout \& W. Rozycki (Eds.), Contrastive rhetoric. Reaching to intercultural rhetoric (pp. 25-41). Ámsterdam: John Benjamins.

Mousavi, A. \& Rauof Moini, M. (2014). A corpus study of shell nuns in published research articles of education. Procedia. Social and Behavioral Sciences, 98, 12821289.

Mur Dueñas, M. P. (2003/2004). Analysing stance in American and Spanish business management RAs: The case of sentence-initial 'retrospective labels'. Journal of English Studies, 4, 137-154.

Picallo, M. C. (1999). La estructura del sintagma nominal: Las nominalizaciones y otros sustantivos con complementos argumentales. En I. Bosque \& V. Demonte (Eds.), Gramática descriptiva de la lengua española. Sintaxis básica de las clases de palabras Vol. 1 (pp. 363-393). Madrid: Espasa Calpe.

Quirk, R., Greenbaun, S., Leech, G. \& Svartvik, J. (1985). A comprehensive grammar of the English language. Essex: Longman.

Real Academia Española (2009). Nueva gramática de la lengua española. Morfología y sintaxis I. Madrid: Espasa Libros.

Schmid, H. J. (2000). English abstract nouns as conceptual shells. From corpus to cognition. Berlín: Mouton de Gruyter. 
Schmid, H. J. (2018). Shell nouns in English. A personal roundup. Caplletra, 64, 109128.

Sinclair, J. (1991). Corpus, concordance, collocation. Oxford: Oxford University Press.

Sinclair, J. (2004). Trust the text. Language, corpus and discourse. Londres: Routledge.

Sinclair, J. (2005). Corpus and text. Basic principles. En M. Wynne (Ed.), Developing linguistic corpora: A guide to good practice (pp. 1-16). Oxford: Oxbow Books.

Swales, J. M. (2005). Attended and unattended 'this' in academic writing: A long and unfinished story. ESP Malasya, 11, 1-15.

Van de Kopple, W. (1985). Some exploratory discourse on metadiscourse. College Composition and Communication, 36(1), 82-93.

Varela Ortega, S. \& Piera, C. (1999). Relaciones entre morfología y sintaxis. En I. Bosque \& V. Demonte (Eds.), Gramática descriptiva de la lengua española. Entre la oración y el discurso. Morfología Vol. 3 (pp. 4367-4422). Madrid: Espasa Calpe.

Winter, E. O. (1977). A clause-relational approach to English texts: A study of some predictive lexical items in written discourse. Instructional Science, 6, 1-92.

Winter, E. O. (1992). The notion of unspecific vs. specific as one way of analysing the information of a fund-raising letter. En W. Mann \& S. A. Thompsn (Eds.), Discourse description: Diverse linguistic analyses of a fund-raising text (pp. 131-169). Ámsterdam: John Benjamins. 


\section{ANEXO}

\section{Listado alfabético de nombres rotuladores en inglés y en español}

\begin{tabular}{|c|c|c|c|c|}
\hline 1ability & 36 conocimientos & 71 funciones & 106 paragraph & 141 scenario \\
\hline 2acción & 37 considerandos & 72 generalization & $\begin{array}{l}107 \text { parámetros - } \\
\text { parameters }\end{array}$ & 142 section $/ \mathrm{s}$ \\
\hline 3advancements & 38 considerations & 73 goal & 108 perspectiva & $\begin{array}{l}143 \text { situación/es - } \\
\text { situation }\end{array}$ \\
\hline 4ajuste - adjustments & 39 contexto & 74 grupos & 109 particularidades & 144 sources \\
\hline $\begin{array}{l}5 \text { alternativa/s - } \\
\text { alternative }\end{array}$ & $\begin{array}{l}40 \text { correlaciones - } \\
\text { correlation/s }\end{array}$ & 75 finding/s & 110 patrón - pattern /s & 145 standard \\
\hline Ganálisis & 41 criterios & 76 hecho & 111 phase & 146 strategy \\
\hline 7approach & 42 dato/s - data & 77 hypothesis/es & 112 phenomenon & 147 study/ies \\
\hline 8 aspecto/s - aspects & 43 description & 78 idea & 113 possibility & 148 system \\
\hline 9assessment/s & 44 details & 79 impacts & 114 potential & 149 técnica - technique \\
\hline 10association/s & 45 determinaciones & 80 improvements & 115 práctica & $\mathbf{1 5 0}$ tendencia - trend \\
\hline 11atributos - attributes & 46 development & $\begin{array}{l}81 \text { información - } \\
\text { information }\end{array}$ & 116 prediction & 151 theory \\
\hline 12increase & $\begin{array}{l}47 \text { diferencia/s - } \\
\text { difference/s }\end{array}$ & 82 instances & 117 premisa & 152 tipo/s - types \\
\hline $\begin{array}{l}\text { 13beneficio/s - } \\
\text { benefits }\end{array}$ & 48 dificultad/es & 83 interactions & 118 principios & 153 trabajo $/ \mathrm{s}$ - work \\
\hline 14cálculo - calculation & 49 dilema & 84 issue/s & $\begin{array}{l}119 \text { problema/s - } \\
\text { problem }\end{array}$ & 154 trade-off \\
\hline 15cambio - changes & $\begin{array}{l}50 \text { discrepancia - } \\
\text { discrepancy/ies }\end{array}$ & 85 kind & 120 problemática & 155 uncertainties \\
\hline 16capacidad & 51 distinction & 86 lack & $\begin{array}{l}121 \text { procedimiento - } \\
\text { procedure/s }\end{array}$ & 156 value \\
\hline 17 caracteres & 52 distribución & $\begin{array}{l}87 \text { limitación - } \\
\text { limitations }\end{array}$ & $\begin{array}{l}122 \text { proceso/s - } \\
\text { process/es }\end{array}$ & 157 variables \\
\hline $\begin{array}{l}18 \text { característica/s - } \\
\text { characteristic/s }\end{array}$ & 53 diversidad & $\mathbf{8 8}$ literature & 123 propiedades & 158 variation \\
\hline 19caracterización & 54 efecto $/ \mathrm{s}-\mathrm{effect} / \mathrm{s}$ & 89 loss & 124 protocolo & 159 view \\
\hline 20 caso $/ \mathrm{s}-$ case $/ \mathrm{s}$ & 55 eficiencia & 90 manera - way/s & 125 punto - point & \\
\hline 21 causas & 56 esquema & 91 marco - framework & 126 purposes & \\
\hline 22 challenge & 57 estado & 92 mecanismo & 127 question/s & \\
\hline 23 classes & $\begin{array}{l}58 \text { estimación - } \\
\text { estimate }\end{array}$ & 93 método & 128 razón/es - reason/s & \\
\hline 24 cita & 59 evidence & $\begin{array}{l}94 \text { metodología/s - } \\
\text { methodology }\end{array}$ & 129 razonamiento & \\
\hline 25 combinación & 60 evolución & $\mathbf{9 5}$ modalidades & 130 rationale & \\
\hline 26 comentario & 61 examples & $\begin{array}{l}96 \mathrm{modelo} / \mathrm{s} \text { - } \\
\mathrm{model} / \mathrm{s}\end{array}$ & $\begin{array}{l}131 \text { recomendaciones - } \\
\text { recommendation }\end{array}$ & \\
\hline 27 comparison/s & 62 exceptions & 97 modification & 132 reduction & \\
\hline 28 complexity & 63 expectations & 98 modo & 133 refinement & \\
\hline 29 components & 64 trials & 99 motivo/s & $\begin{array}{l}\mathbf{1 3 4} \text { relación - } \\
\text { relationship }\end{array}$ & \\
\hline $\begin{array}{l}30 \text { comportamiento/s } \\
\text { - behaviour }\end{array}$ & 65 experiment & 100 necesidad & 135 requirement & \\
\hline 31 compromises & $\begin{array}{l}66 \text { explicación - } \\
\text { explanation }\end{array}$ & 101 note & $\begin{array}{l}136 \text { respuesta/s - } \\
\text { response/s }\end{array}$ & \\
\hline $\begin{array}{l}32 \text { concepto/s - } \\
\text { concepts }\end{array}$ & 67 facets & $\begin{array}{l}102 \text { objetivo/s - } \\
\text { objetive/s }\end{array}$ & 137 restrictions & \\
\hline 33 concerns & 68 factor/es - factor/s & $\begin{array}{l}\text { s } 103 \text { observación/es - } \\
\text { observation/s }\end{array}$ & 138 resultado/s - result/s & \\
\hline $\begin{array}{l}34 \text { conclusiones - } \\
\text { conclusion/s }\end{array}$ & 69 fin - end & 104 operation & 139 rules & \\
\hline 35 condición/es - & 70 formulation & 105 options & 140 salto & \\
\hline
\end{tabular}




\section{NOTAS}

${ }^{1}$ Este trabajo es una reformulación de un capítulo de la tesis doctoral elaborada por Verónica Muñoz y dirigida por Guiomar Ciapuscio, aprobada el 4 de julio de 2017 (Facultad de Lenguas, Universidad Nacional de Córdoba).

${ }^{2}$ A fin de evitar problemas terminológicos empleamos el término 'nombres rotuladores'.

${ }^{3}$ Agricultural Systems, Applied Soil Ecology, Field Crops Research, y Plant and Soil - Agriscientia, Ciencia del Suelo, Revista de Investigaciones Agropecuarias (RIA), y Revista de la Facultad de Ciencias Agrarias.

${ }^{4}$ Descartamos casos en los que los nombres como way y modo ocurrían en expresiones gramaticalizadas que funcionan como construcciones conectivas (por ej., de esta manera). Al estar integrados a expresiones fijas, en estos entornos sintácticos los nombres no tienen carácter nominal pleno y no funcionan con autonomía. La secuencia supone una única elección (Francis, 1994; López Samaniego, 2011; Flowerdew, 2015; Flowerdew \& Forest, 2015). 